\title{
Interpreting Cytokinin Action as Anterograde Signaling and Beyond
}

\author{
Yoshihisa Ikeda1*, David Zalabák², Ivona Kubalová ${ }^{3}$, Michaela Králová1, \\ Wolfram G. Brenner ${ }^{4}$ and Mitsuhiro Aida ${ }^{5}$
}

\begin{abstract}
${ }^{1}$ Centre of the Region Haná for Biotechnological and Agricultural Research, Czech Advanced Technology and Research Institute, Palacký University, Olomouc, Czechia, ${ }^{2}$ Laboratory of Growth Regulators, Palacky University and Institute of Experimental Botany AS CR, Olomouc, Czechia, ${ }^{3}$ Leibniz Institute of Plant Genetics and Crop Plant Research (IPK), Gatersleben, Germany, ${ }^{4}$ General and Applied Botany, Institute of Biology, Universität Leipzig, Leipzig, Germany, ${ }^{5}$ International Research Organization for Advanced Science and Technology (IROAST), Kumamoto University, Kumamoto,
\end{abstract} Japan

\section{OPEN ACCESS}

Edited by:

Anna N. Stepanova,

North Carolina State University,

United States

Reviewed by:

Venugopala Reddy Gonehal, University of California, Riverside,

United States

Yuling Jiao,

Institute of Genetics

and Developmental Biology, Chinese

Academy of Sciences, China

Cristiana T. Argueso,

Colorado State University,

United States

*Correspondence:

Yoshihisa Ikeda

yoshihisa.ikeda@upol.cz

Specialty section:

This article was submitted to

Plant Physiology,

a section of the journal

Frontiers in Plant Science

Received: 13 December 2020 Accepted: 08 March 2021

Published: 29 March 2021

Citation:

Ikeda Y, Zalabák D, Kubalová I, Králová M, Brenner WG and Aida M (2021) Interpreting Cytokinin Action as

Anterograde Signaling and Beyond.

Front. Plant Sci. 12:641257.

doi: 10.3389/fp/s.2021.641257
Among the major phytohormones, the cytokinin exhibits unique features for its ability to positively affect the developmental status of plastids. Even early on in its research, cytokinins were known to promote plastid differentiation and to reduce the loss of chlorophyll in detached leaves. Since the discovery of the components of cytokinin perception and primary signaling, the genes involved in photosynthesis and plastid differentiation have been identified as those directly targeted by type-B response regulators. Furthermore, cytokinins are known to modulate versatile cellular processes such as promoting the division and differentiation of cells and, in concert with auxin, initiating the de novo formation of shoot apical meristem (SAM) in tissue cultures. Yet how cytokinins precisely participate in such diverse cellular phenomena, and how the associated cellular processes are coordinated as a whole, remains unclear. A plausible presumption that would account for the coordinated gene expression is the tight and reciprocal communication between the nucleus and plastid. The fact that cytokinins affect plastid developmental status via gene expression in both the nucleus and plastid is interpreted here to suggest that cytokinin functions as an initiator of anterograde (nucleus-to-plastid) signaling. Based on this viewpoint, we first summarize the physiological relevance of cytokinins to the coordination of plastid differentiation with de novo shoot organogenesis in tissue culture systems. Next, the role of endogenous cytokinins in influencing plastid differentiation within the SAM of intact plants is discussed. Finally, a presumed plastid-derived signal in response to cytokinins for coupled nuclear gene expression is proposed.

Keywords: anterograde signaling, cytokinin, chloroplast, organelle communication, retrograde signaling, shoot apical meristem, tissue culture, WUSCHEL

\section{INTRODUCTION}

Since their discovery over 60 years ago, cytokinins (CKs), in concert with auxin, have been shown to promote cell division and shoots in an in vitro tissue culture system (Miller et al., 1955; Skoog and Miller, 1957), while a treatment of kinetin alone mitigated the chlorophyll losses in detached Xanthium leaves (Richmond and Lang, 1957). Although dark-grown seedlings treated with CKs 
exhibited de-etiolated traits, it appears that CKs and light signaling operate independently or sequentially via partially overlapping pathways (Chory et al., 1994). Myriad studies confirm the positive effects of CKs on plastids' functioning: protecting them from high light-induced damage, and preventing chlorophyll degradation under dark conditions (Cortleven and Schmülling, 2015). Indeed, the expression of genes encoding a subset of enzymes in the chlorophyll biosynthesis pathway and those encoding the multiple subunits of the lightharvesting complex (LHC) were augmented by a CK treatment (Cortleven et al., 2016).

All plastids present in aboveground tissues are derived from those in the central zone $(\mathrm{CZ})$ of the shoot apical meristem (SAM). The developmental status of plastids in the SAM was considered proplastid (Lopez-Juez and Pyke, 2005; Fleming, 2006; Sakamoto et al., 2008) until recently, when Charuvi et al. (2012) revealed that it varied in the shoot apex, depending on the position or layer they were located: plastids in the CZ and peripheral zone (PZ) of the L1 and L3 could partially develop thylakoid membranes. Several studies demonstrated the CZ of L3 (organizing center/OC; also referred to as the rib meristem) is the site for endogenous cytokinin perception (Gordon et al., 2009; Zürcher et al., 2013; Gruel et al., 2016; Snipes et al., 2018). Despite the fact that plastids in endogenous CK-rich domains are poorly developed, a highresolution gene expression map in the SAM uncovered greater expression of genes encoding the photosystem I (PSI) LHC $\mathrm{A}$ and its subunit proteins, as well as photosystem II (PSII) components and its subunit protein in the L3, by $>1.5$-fold (relative to other domains) (Yadav et al., 2014). These findings suggest the $\mathrm{CZ}$ of the shoot apex provides a unique system for investigating the impact of endogenous CKs in the early phase of plastid development.

In this perspective article, we attempt to interpret the action of $\mathrm{CK}$ as anterograde signaling. As proposed in prior reviews, the term "anterograde signaling" refers to the process whereby nucleus-derived regulators that transmit information express proteins to coordinate the expression of genes in the nucleus and plastids at multiple levels (Pesaresi et al., 2007; Kleine et al., 2009; Jung and Chory, 2010; Berry et al., 2013). In this way, multiprotein complex machineries, such as photosystems, cytochrome b6/f or ATP synthase, are optimally assembled (Kleine et al., 2009). We begin by focusing on how exogenous and endogenous CKs affect the expression of genes encoding tetrapyrrole biosynthesis, a fundamental metabolic pathway in all living organisms (Figure 1A; Tanaka et al., 2011), and then summarizing these effects. Although all genes involved in tetrapyrrole biosynthesis are in the nuclear genome, we scrutinized them because some were found to be CK-responsive (reviewed by Cortleven and Schmülling, 2015). Lastly, based on the results obtained from an in vitro tissue culture system (Kubalová et al., 2019), the plausible involvement of retrograde signaling in modulating expression of the nuclear gene WUSCHEL (WUS), a master regulator of stem cell niche, is explored.

\section{INITIATION OF ANTEROGRADE REGULATION BY CKs}

An exogenous CK treatment can greatly impact plastids' development and functioning (Cortleven and Schmülling, 2015). Gene expression analyses have corroborated this, by uncovering upregulated plastid-encoded genes upon CK treatment in Arabidopsis and barley (Brenner et al., 2005; Zubo et al., 2008; Danilova et al., 2017a,b; Andreeva et al., 2020). In Arabidopsis, upregulated plastid genes include components of cytochrome b6/f (petA) and PSII (PsbG, psbA, and psbl) (Brenner et al., 2005). Both machineries consist of protein complexes encoded in the nucleus and plastids. Additionally, CK indirectly affects plastids' gene expression by upregulating nuclear genes that encode components of their transcription machinery (i.e., phage-type RNA-polymerases, sigma factors, and plastid RNA polymeraseassociated proteins; Danilova et al., 2017a; Andreeva et al., 2020). These results imply that CK-coordinated gene expression activity in the nucleus and plastid provides appropriate information for balancing metabolic flows in plastids and cellular functions.

\section{RELATIONSHIP BETWEEN PLASTID STATUS IN THE SAM AND CK SIGNALING IN TISSUE CULTURE}

The in vitro tissue culture technique has been employed to study pluripotency. In such a system, de novo organogenesis is manipulated by a defined cytokinin-to-auxin ratio in cultured medium. As Figure 1B (top) illustrates, this widely used method has two sequential steps (Valvekens et al., 1988). The first involves pre-incubation of explants on an auxin-rich callusinducing medium (CIM), to induce a mass of growing cells, termed a callus. The second step promotes the greening of foci when these induced calli are cultured on shoot-inducing medium (SIM) to which a high cytokinin-to-auxin ratio has been applied. The underlying mechanisms of these processes are detailed in several recent review articles (Shin et al., 2020; Xu and $\mathrm{Hu}, 2020)$. The external application of CKs induces WUS expression likely via direct activation by type B Arabidopsis Response Regulators (B-ARRs) (Dai et al., 2017; Meng et al., 2017; Zhang et al., 2017; Zubo et al., 2017; Xie et al., 2018); albeit a high CK concentration is required (Gordon et al., 2009). The tight linkage between CK-stimulated WUS expression and de novo shoot regeneration has been corroborated by genetic studies. Loss-of-function mutations in WUS compromised de novo shoot regeneration (Gordon et al., 2007; Chatfield et al., 2013; Zhang et al., 2017). Mutants with decreased CK perception were characterized by poor shoot regeneration (Ueguchi et al., 2001; Nishimura et al., 2004; Riefler et al., 2006; Pernisova et al., 2018). By contrast, root explants of CK-hypersensitive mutantsckh1 defective in the gene encoding TATA-box binding protein (TBP)-associated factor TAF12-like protein, or ckh2/pkl (pickle) defective in gene encoding CHD3 class of SWI/SNF chromatin remodeling factor-promoted tissue greening on CIM culture (Furuta et al., 2011) and developed de novo shoots more rapidly 
A

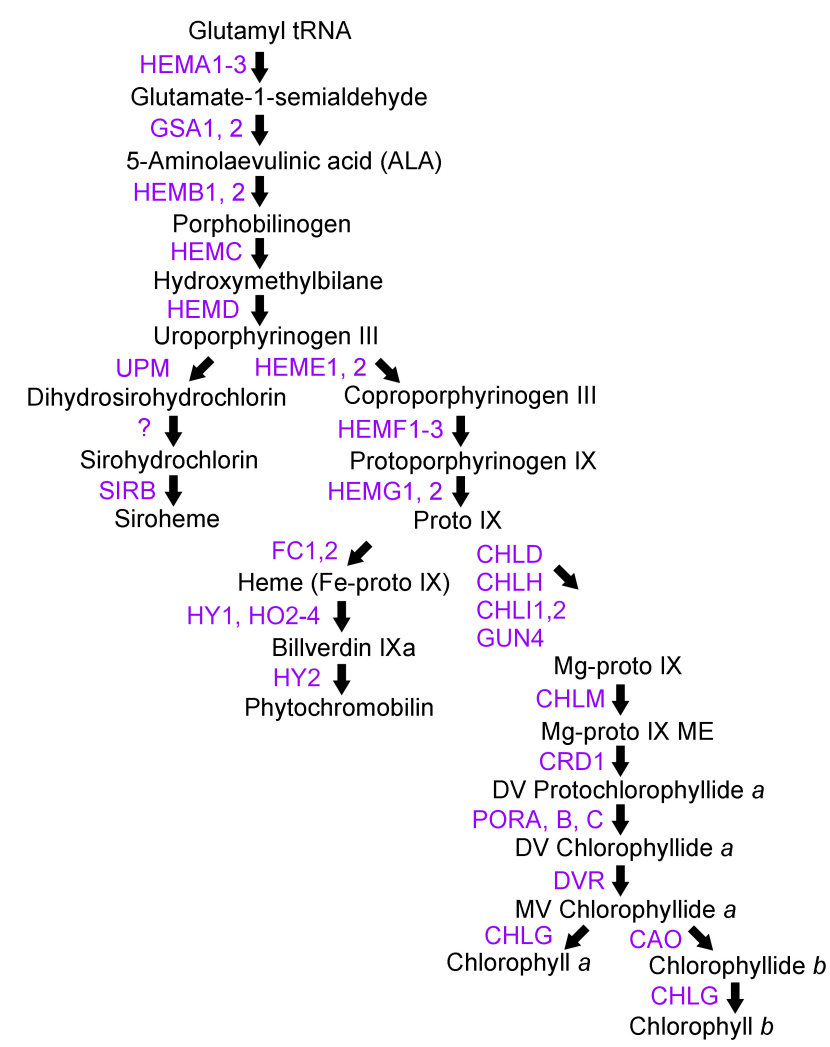

B

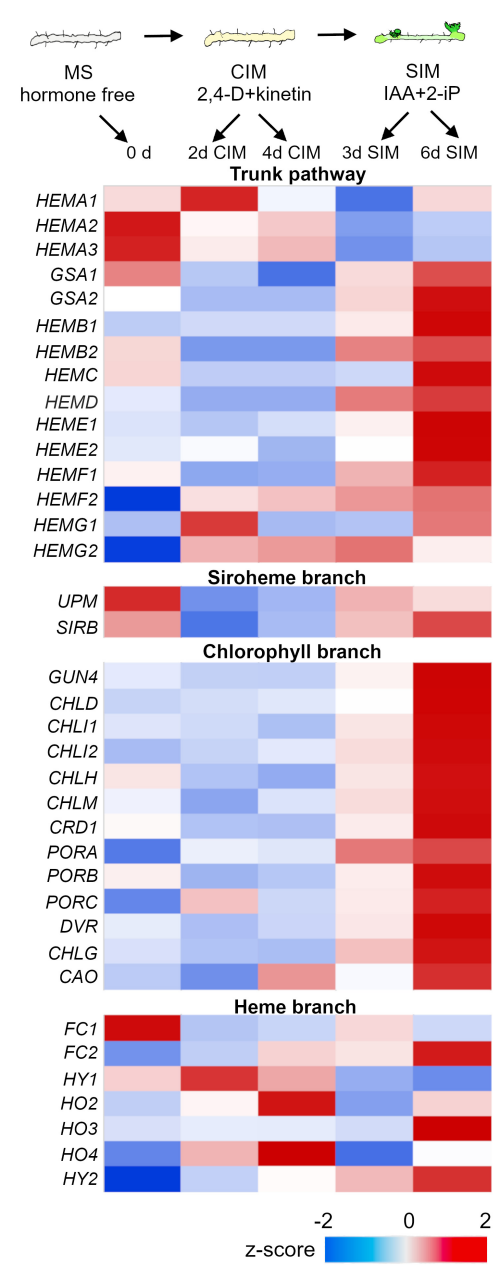

C

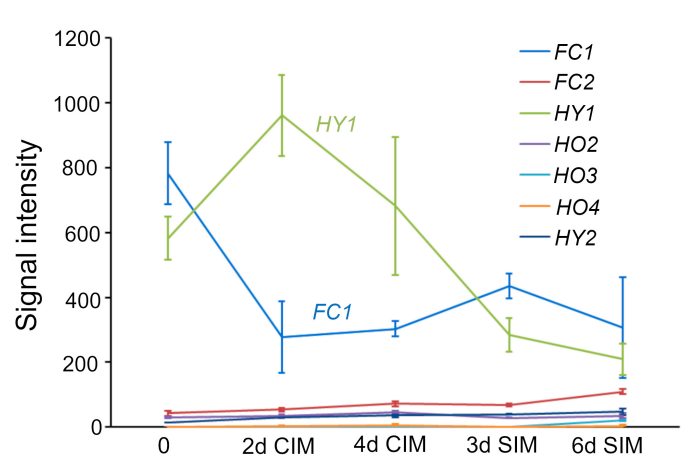

D

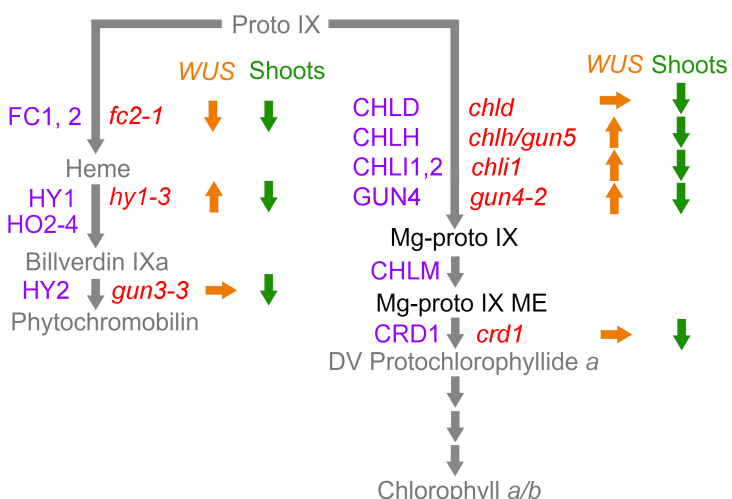

Chlorophyll $a / b$

FIGURE 1 | The relationship between the tetrapyrrole biosynthesis pathway and cytokinin-mediated de novo shoot apical meristem (SAM) development in tissue culture. (A) Tetrapyrrole biosynthesis pathway. Arrows indicate enzymatic reactions. Abbreviations of enzyme names are depicted in purple. (B) Schematic morphological representation of excised root explants cultured on MS (Murashige and Skoog medium), CIM (callus-inducing medium), and SIM (shoot-inducing medium) (top), and the expression profiles of genes involved in tetrapyrrole biosynthesis at indicated time points (day) of the indicated culture medium (bottom). Relative expression levels (z-scores) are displayed using the color code (blue to red). All the data came from the Arabidopsis eFP browser (http://bar.utoronto.ca/efp_arabidopsis/cgi-bin/efpWeb.cgi?dataSource = Regeneration). (C) Signal intensity of genes involved in the heme branch. (D) Summary of relative expression levels of WUS (orange arrows) and shoot regeneration efficiency (green arrows) in root explants of tetrapyrrole mutants cultured on SIM. Up, down, and right arrows indicate an increased WUS transcript level, decreased WUS transcript level or compromised shoot regeneration, or statistically insignificant changes in the WUS transcript level, respectively. The names of enzymes and corresponding mutants are, respectively, depicted in purple and red. 
and more frequently during the course of SIM incubation. The shoot regeneration efficiencies in the mutants with altered CK perception coincided well with their WUS expression levels (Kubalová and Ikeda, 2017; Kubalová et al., 2019).

\section{CHANGES TO TETRAPYRROLE BIOSYNTHESIS GENE EXPRESSION AT THE ONSET OF SHOOT REGENERATION}

As expected from the greening of a tissue phenotype of root explants cultured on SIM (Figure 1B top), analysis of a previously reported microarray expression profile (eFP browser) ${ }^{1}$ (Che et al., 2002) confirms the increased expression of genes involved in the pathway leading to the chlorophyll $a / b$ (trunk pathway and chlorophyll branch) (Figure 1B). The exception was the HEMA genes, whose products catalyze the initial step in the trunk tetrapyrrole pathway (Kumar and Söll, 2000), their transcripts were substantially reduced when root explants were transferred from CIM onto SIM. This is very intriguing because HEMA1, encoding GLUTAMYL-tRNA-REDUCTASE (GluTR), is mainly expressed in photosynthetic tissues, regulated by light through phytochromes (Papenbrock et al., 1999; McCormac et al., 2001), and induced by CK when applied to dark-grown seedlings (Cortleven et al., 2016). In stark contrast to the increased expression of genes involved in the chlorophyll branch, the expression of genes involved in the heme branch did not respond well to SIM incubation. Rather, transcripts of $L O N G$ HYPOCOTYL 1 (HY1)/HEME OXYGENASE 1 (HO1) declined over time (Figure 1B). The expression levels of $\mathrm{HO} 3$ and HO4 increased, but their contribution seems minor because the signal intensities of their transcripts are negligible when compared to that of HY1 (Figure 1C). Although no quantification results of tetrapyrrole molecules during SIM incubation are currently available, we may reasonably speculate that this reduced HEMA expression is due to an insufficient availability of phytochromobilin, the chromophore of phytochromes. It has been repeatedly documented that a wide range of phytochromemediated light responses become impaired in hyl plants; this suggests light-induced HEMA1 expression is impaired when the HY1's transcription diminished under the SIM incubation. Notably, HEMA enzymatic activity is attenuated by heme accumulation, in a feedback mechanism (Pontoppidan and Kannangara, 1994), and HEMA proteins are destabilized by heme (Richter et al., 2019). Nevertheless, the HEMA transcripts in hyl are comparable to those in the wild type (Goslings et al., 2004). Collectively, those findings suggest that, although expression of the rate-limiting HEMA1 is repressed, chlorophyll $a / b$ can readily accumulate during SIM incubation (as evinced by tissue greening). Conversely, the heme branch is likely to be attenuated by decreased expression of $H Y 1$, implying that heme accumulation occurs when de novo SAM is being initiated in the CK-enriched culture. Further analysis to determine the

${ }^{1}$ http://bar.utoronto.ca/efp_arabidopsis/cgi-bin/efpWeb.cgi?dataSource= Regeneration tetrapyrrole molecule contents during cytokinin-stimulated de novo SAM emergence is required.

\section{WUS EXPRESSION IS UNCOUPLED FROM DE NOVO SAM FORMATION IN MUTANTS DEFECTIVE IN TETRAPYRROLE BIOSYNTHETIC PATHWAY}

Microarray results also validate the greater expression of meristem-related genes upon CK-rich SIM incubation (Che et al., 2002). B-ARRs encoding GARP transcription factors directly activated WUS expression (Meng et al., 2017; Zhang et al., 2017; Xie et al., 2018). Loss-of-function mutations of tetrapyrrole biosynthesis provide a valuable tool to study the relationship between tissue greening and shoot regeneration. All the mutants defective in the heme branch (protoporphyrin ix ferrochelatase 2/fc2, hy1, genome-uncoupled 3/gun3), and chlorophyll branch (gun4, Mg-chelatase d/chld, chlh/gun5, chli1, copper response deficient $1 /$ crd1) exhibiting reduced chlorophyll contents resulted in decreased shoot regeneration efficiency (Kubalová et al., 2019); hence, proper chlorophyll contents are required for the acquisition of de novo SAM competence or shoot regeneration efficiency. Unexpectedly, of eight tetrapyrrole biosynthesis mutants with substantially compromised shoot regeneration efficiency, in four (hy1-3, gun4-2, chlh/gun5, chli1) the expression level of WUS significantly increased (Kubalová et al., 2019). This suggests tetrapyrrole intermediates influence nuclear-encoded WUS expression. Notably, WUS was expressed most in the hy1-3 mutant despite its shoot regeneration efficiency being among the lowest (Kubalová et al., 2019). In summary, WUS expression in hy1-3, gun4-3, chlh, and chli1 mutants is uncoupled from shoot regeneration efficiency (Figure 1D), suggesting that, in response to the altered plastid developmental status stimulated by the high cytokinin-to-auxin ratio in SIM, plastid-to-nucleus communication occurs to fine-tune nuclear WUS expression during de novo shoot organogenesis.

\section{ALTERED PLASTID DIFFERENTIATION STATUS IN THE SAM}

All aboveground tissues arise from the SAM, which contains stem cells in its CZ. The plastids in SAM were once considered proplastids (Lopez-Juez and Pyke, 2005; Fleming, 2006; Sakamoto et al., 2008). Despite that prevailing concept, sophisticated morphological analyses of plastids in Arabidopsis SAM revealed their developmental status was not necessarily null and, depending on their position within the SAM, it had dynamic features: plastids underwent different developmental processes that could lead to either the acquisition or loss of thylakoid membranes (Charuvi et al., 2012). The outermost layer L1 (protoderm) gives rise to the epidermis whose photosynthetic activity is lost once leaves differentiate. Yet, plastids within L1 layer's cells of the SAM (both in CZ and PZ) developed partially 

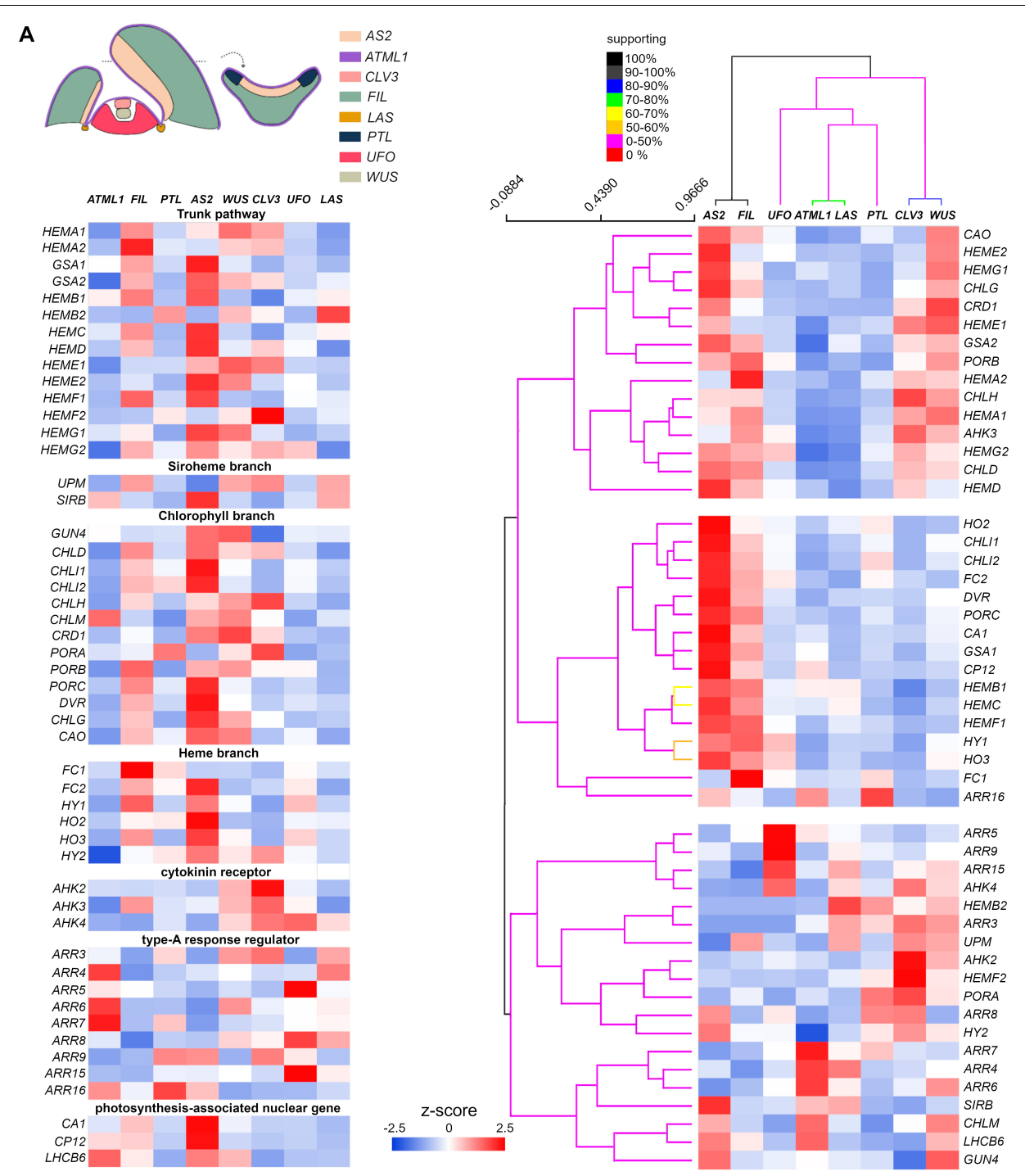

B

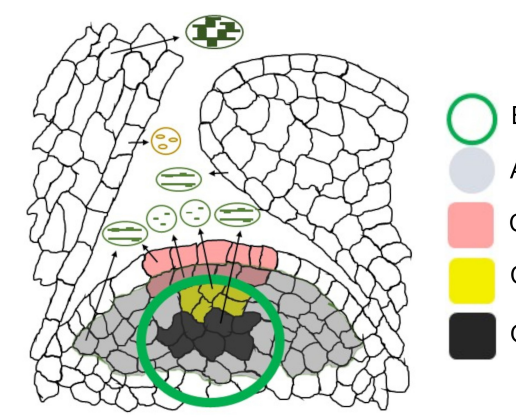

Endogenous CK-response site

Absent from chlorophyll autofluorescence

Central zone (L1 and L2)

Central zone overlapping with $\mathrm{OC}$

Organizing center (OC)

FIGURE 2 | SAM as a unique tissue to study endogenous cytokinin (CK) action on early developmental status of plastids. (A) Expression of genes involved in tetrapyrrole biosynthesis, with CK-related and light-responsive genes serving as references for CK or light perception, respectively. The same sets of genes are arranged according to the functional category (left) and to their expression patterns deduced from the clustering analysis (right). Genes and shoot apex domains shown in the left panel were clustered for similarity, in terms of their gene expression pattern, by the Support Tree algorithm implemented in TIGR MeV4 (Saeed et al., 2003), using $n=100$ iterations of bootstrapping both genes and shoot apex domains. Relative expression levels determined by the $z$-scores are color-coded (in blue to red). Percentage of support for the clustering analysis is depicted according to a color intensity gradient [from red (0\%) to black (100\%)]. All the data came from the Arabidopsis eFP browser (http://bar.utoronto.ca/efp_arabidopsis/cgi-bin/efpWeb.cgi?dataSource = Shoot_Apex), except HEMA3, HO4, and ARR17 (Tian et al., 2019). (B) Summary of plastid differentiation status in cells differentially positioned in the shoot apex. The plastid status in various types of cells is illustrated, with those cells lacking autofluorescence highlighted in gray [adapted from Charuvi et al., 2012)]. Cells able to respond to endogenous CKs (as revealed by TCS reporters) are indicated by a green circle (Zürcher et al., 2013). The L1 and L2 cells within the CZ of SAM are highlighted in orange; the cells in L3 overlapping with the organizing center $(\mathrm{OC})$ appear in yellow. Cells comprising the $\mathrm{OC}$ are shown in black. 
differentiated thylakoid networks. The subepidermal layer L2, located below L1, is the progenitor of outer mesophyll tissue. Despite the fact that the L2 is the major source of photosynthetic tissues, the plastids in the CZ of L2 persist in the most undeveloped state, devoid of thylakoid networks characteristic of proplastids. By contrast, plastids in the PZ cells of L2 develop thylakoid membranes. Beneath them is L3, a multilayer where a small group of cells comprise an OC whose cell fate remains undifferentiated. Inner tissues, such as inner mesophyll and vascular cells, originate from the L3. The differentiation status of plastids in L3's uppermost layer in CZ is similar to that of L2 in CZ (proplastids), whereas plastids in L3's inner layers are closer to maturity, having partially developed thylakoid networks.

So the developmental status of plastids within the SAM seems under internal control, and light signaling does not appear to figure prominently because the plastids can better develop thylakoid networks in the inner L3 than in L2. Expression of a CK-reporter gene, TCS (Two-component Signaling Sensor) and its derivative TCSn, indicated that the primary CKsignaling response was confined within the CZ of L3 (Gordon et al., 2009; Zürcher et al., 2013; Snipes et al., 2018). The majority of molecules participating in primary CK signalingARABIDOPSIS HISTIDINE KINASE/AHK receptors, AHP phosphotransmitters, and B-ARRs-are expressed to some extent in Arabidopsis SAM (Nishimura et al., 2004; Gordon et al., 2009; Gruel et al., 2016). Three CK receptors, AHK2-AHK4, are predominantly expressed in L3 cells (Gruel et al., 2016) and WUS-expressing cells coincide well with AHK4-expressing cells (Gordon et al., 2009). Recently, using several tissuespecific promoters, Tian et al. (2019) determined the differential expression pattern of translating RNAs in differing shoot domains, termed "domain-specific translatome map." To gain insight into CK action's relation to plastid differentiation status in the SAM, we extracted expression profiles of genes involved in tetrapyrrole biosynthesis, whose results were standardized and conveyed here using $z$-scores (Figure 2A). Although for three $\mathrm{CK}$ receptor genes, their expression is reportedly absent in L1 (Gruel et al., 2016), their abundant expression in domains of CLV3 (CLAVATA 3) and WUS agree well with those of high-CK responses revealed by expression of the TCS reporters (Figure $\mathbf{2 A}$ ). These results strongly suggest the SAM, especially the CZ of L3, is the active site for the perception of endogenous CKs. Nevertheless, those plastids within L2 and L3 remained the least differentiated; in fact, chlorophyll autofluorescence in the corresponding region was below the detection threshold (Figure 2B; Charuvi et al., 2012; Yadav et al., 2019). Unlike for poorly developed plastid conditions in the CLV3 or WUS domain, several tetrapyrrole biosynthesis genes (HEMA1, HEME1, HEMF2, CHLH, CRD1, and $P O R A$ ) exhibited the highest transcript levels (Figure 2A), notwithstanding the fact that the expression of HEMA1, CHLH, and $C R D 1$ was predominantly induced by light in photosynthetic tissues (Matsumoto et al., 2004). These results suggest that a handful of genes involved in tetrapyrrole biosynthesis are not only regulated by light but also by intrinsic signal(s). A plausible candidate for this might be CKs or the interplay between them and other hormones. It appears that upregulation of the above genes contributes little or negligibly to chlorophyll biosynthesis in the CZ of L2 and L3, since chlorophyll autofluorescence is undetectable in these layers (Figure 2B; Charuvi et al., 2012; Yadav et al., 2019). The expression of genes involved in the heme branch is generally low in both CLV3 and WUS domains, especially that of $H Y 1$ (lowest in the CLV3 domain; Figure 2A), indicative of heme accumulation. To gain further insight into the negative relationship between HYl's expression and CK signaling, those genes involved in CKs' perception and response, as well as light-responsive genes, were analyzed by clustering. These results confirmed that $H Y 1$ fell into a cluster distinct from that harboring the two $\mathrm{CK}$ receptors (AHK2 and $A K H 4$ ) and eight of nine A-ARRs (Figure 2A, right panel). Studying the developmental status of plastids in cells in the CZ of L2 and the uppermost layers of L3 in the CZ, by manipulating endogenous CK levels, would provide a new opportunity to reveal as-of-yet-unknown CK functions in early plastid development. In two different systems, for de novo organogenesis in tissue culture and the shoot apices of light-grown seedlings, the HY1 expression levels are negatively correlated with exogenous CK or endogenous CK-rich domains (Figures 1, 2A). Although further analyses are required to uncover a negative correlation between the two mentioned above, it is likely that, in response to CK as an initiator of anterograde signaling, the plastid-to-nucleus communication happens predominantly in meristematic cells where endogenous CK maxima are established.

\section{CONCLUSION AND PERSPECTIVE}

Bioactive CKs are molecules perceived by the CHASE domain of CK receptors, for which the primary two-component multistep phosphorelay represents the output module able to regulate gene expression. Given the fact that exogenous CK application to dark-grown plants promotes etioplast-to-chloroplast transition, probably via altered gene expression both in the nucleus and plastids, CKs could positively regulate anterograde signaling to promote plastid differentiation. It appears that the particular developmental and differentiation status affects the sensitivity to $\mathrm{CK}$ in plant cells. We propose the CZ of SAM as a promising site to investigate endogenous $\mathrm{CK}$ action influencing early plastid development (Figure 2B). An alternative motive for this approach is to gain better insight into the coordinated regulation of stem cell determination vis-à-vis the plastids' developmental status. The molecular regulatory mechanism underlying diminished expression of HY1 by CK is not entirely clear. The lower auxin-to-cytokinin ratio in SIM may be responsible for declines in $H Y 1$ expression because $H Y 1$ is induced by auxin and acts downstream of it (Ma et al., 2014). Besides, an auxin minimum is known to exist in the OC of SAM (Shi et al., 2018). Either way, genetic studies have revealed the overlooked contribution of the heme branch (Kubalová et al., 2019). Although tetrapyrrole biosynthesis is controlled under complex regulation, it is reasonable to argue that substrates accumulate when the catalytic enzymatic step is blocked. Since WUS expression in $f_{c} 2-1$ is evidently opposite to that in hy1-3, gun4-3, chlh, and chli1 (Figure 1D), it is 
tempting to speculate that heme, whose synthesis is blocked in $f_{c} 2-1$ but which accumulates in $h y 1-3$, is the plausible candidate molecule modulating WUS expression. It goes without saying that the alternative interpretations remain open to account for this. Interestingly, hyl mutant seedlings accumulate substantially elevated level of endogenous jasmonates by unknown mechanisms and, as a result, jasmonic acid responses are constitutively active (Zhai et al., 2007). Alternatively, the decreased carbon monoxide, a byproduct of heme oxygenase enzymatic reaction, may come into play (Xuan et al., 2008; Ma et al., 2014).

Beyond its diverse functions to serve as cofactors of hemoproteins in various organelles (Layer et al., 2010), several recent studies pose heme as a retrograde signal that is involved in various physiological regulations (Mense and Zhang, 2006; Von Gromoff et al., 2008). Besides, biochemical evidence corroborates this, i.e., half of the identified heme-binding proteins localize in the nucleus in plants (Shimizu et al., 2020). Measuring or monitoring heme content during de novo organogenesis and in the CZ of the SAM of intact plants will provide us with a new insight into the role of the heme branch in nuclear gene expression.

\section{DATA AVAILABILITY STATEMENT}

Publicly available datasets were analyzed in this study. This data can be found here: http://bar.utoronto.ca/efp_arabidopsis/cgi-

\section{REFERENCES}

Andreeva, A. A., Vankova, R., Bychkov, I. A., Kudryakova, N. V., Danilova, M. N., Lacek, J., et al. (2020). Cytokinin-regulated expression of Arabidopsis thaliana pap genes and its implication for the expression of chloroplast-encoded genes. Biomolecules 10, 1-18. doi: 10.3390/biom 10121658

Berry, J. O., Yerramsetty, P., Zielinski, A. M., and Mure, C. M. (2013). Photosynthetic gene expression in higher plants. Photosynth Res. 117, 91-120. doi: 10.1007/s11120-013-9880-8

Brenner, W. G., Romanov, G. A., Köllmer, I., Bürkle, L., and Schmülling, T. (2005). Immediate-early and delayed cytokinin response genes of Arabidopsis thaliana identified by genome-wide expression profiling reveal novel cytokinin-sensitive processes and suggest cytokinin action through transcriptional cascades. Plant J. 44, 314-333. doi: 10.1111/j.1365-313X.2005.02530.x

Charuvi, D., Kiss, V., Nevo, R., Shimoni, E., Adam, Z., and Reich, Z. (2012). Gain and loss of photosynthetic membranes during plastid differentiation in the shoot apex of Arabidopsis. Plant Cell 24, 1143-1157. doi: 10.1105/tpc.111. 094458

Chatfield, S. P., Capron, R., Severino, A., Penttila, P. A., Alfred, S., Nahal, H., et al. (2013). Incipient stem cell niche conversion in tissue culture: using a systems approach to probe early events in WUSCHEL-dependent conversion of lateral root primordia into shoot meristems. Plant J. 73, 798-813. doi: 10.1111/tpj. 12085

Che, P., Gingerich, D. J., Lall, S., and Howell, S. H. (2002). Global and hormoneinduced gene expression changes during shoot development in Arabidopsis. Plant Cell 14, 2771-2785. doi: 10.1105/tpc.006668

Chory, J., Reinecke, D., Sim, S., Washburn, T., and Brenner, M. (1994). A role for cytokinins in de-etiolation in Arabidopsis. det Mutants have an altered response to cytokinins. Plant Physiol. 104, 339-347. doi: 10.1104/pp.104.2.339

Cortleven, A., Marg, I., Yamburenko, M. V., Schlicke, H., Hill, K., Grimm, B., et al. (2016). Cytokinin regulates the etioplast-chloroplast transition through the two-component signaling system and activation of chloroplast-related genes. Plant Physiol. 172, 464-478. doi: 10.1104/pp.16.00640
bin/efpWeb.cgi?dataSource=Regeneration; http://bar.utoronto. ca/efp_arabidopsis/cgi-bin/efpWeb.cgi?dataSource=Shoot_ Apex.

\section{AUTHOR CONTRIBUTIONS}

YI conceived the study's idea. YI, IK, MK, DZ, WB, and MA wrote the manuscript. WB conducted the clustering analysis. All authors contributed to the article and approved the submitted version.

\section{FUNDING}

This work was supported by the Czech Science Foundation grants (GACR17-23702S and GACR 18-23972Y) and IROAST research unit on plant stem cells and regeneration.

\section{ACKNOWLEDGMENTS}

We acknowledge the Czech Science Foundation grants (GACR17-23702S and GACR 18-23972Y) and IROAST research unit on plant stem cells and regeneration, based at Kumamoto University and Véronique Bergougnoux-Fojtik for critical reading of the manuscript.

Cortleven, A., and Schmülling, T. (2015). Regulation of chloroplast development and function by cytokinin. J. Exp. Bot. 66, 4999-5013. doi: 10.1093/jxb/erv132

Dai, X., Liu, Z., Qiao, M., Li, J., Li, S., and Xiang, F. (2017). ARR12 promotes de novo shoot regeneration in Arabidopsis thaliana via activation of WUSCHEL expression. J. Integr. Plant Biol. 59, 747-758. doi: 10.1111/jipb.12567

Danilova, M. N., Doroshenko, A. S., Zabrodin, D. A., Kudryakova, N. V., Oelmüller, R., and Kusnetsov, V. V. (2017a). Cytokinin membrane receptors modulate transcript accumulation of plastid encoded genes. Russ. J. Plant Physiol. 64, 301-309. doi: 10.1134/S1021443717030062

Danilova, M. N., Kudryakova, N. V., Doroshenko, A. S., Zabrodin, D. A., Rakhmankulova, Z. F., Oelmüller, R., et al. (2017b). Opposite roles of the Arabidopsis cytokinin receptors AHK2 and AHK3 in the expression of plastid genes and genes for the plastid transcriptional machinery during senescence. Plant Mol. Biol. 93, 533-546. doi: 10.1007/s11103-016-0580-6

Fleming, A. (2006). Metabolic aspects of organogenesis in the shoot apical meristem. J. Exp. Bot. 57, 1863-1870. doi: 10.1093/jxb/erj178

Furuta, K., Kubo, M., Sano, K., Demura, T., Fukuda, H., Liu, Y. G., et al. (2011). The CKH2/PKL chromatin remodeling factor negatively regulates cytokinin responses in Arabidopsis calli. Plant Cell Physiol. 52, 618-628. doi: 10.1093/pcp/ pcr022

Gordon, S. P., Chickarmane, V. S., Ohno, C., and Meyerowitz, E. M. (2009). Multiple feedback loops through cytokinin signaling control stem cell number within the Arabidopsis shoot meristem. Proc. Natl. Acad. Sci. U.S.A. 106, 16529-16534. doi: 10.1073/pnas.0908122106

Gordon, S. P., Heisler, M. G., Reddy, G. V., Ohno, C., Das, P., and Meyerowitz, E. M. (2007). Pattern formation during de novo assembly of the Arabidopsis shoot meristem. Development 134, 3539-3548. doi: 10.1242/dev.0 10298

Goslings, D., Meskauskiene, R., Kim, C., Lee, K. P., Nater, M., and Apel, K. (2004). Concurrent interactions of heme and FLU with Glu tRNA reductase (HEMA1), the target of metabolic feedback inhibition of tetrapyrrole biosynthesis, in darkAnd light-grown Arabidopsis plants. Plant J. 40, 957-967. doi: 10.1111/j.1365313X.2004.02262.x 
Gruel, J., Landrein, B., Tarr, P., Schuster, C., Refahi, Y., Sampathkumar, A., et al. (2016). An epidermis-driven mechanism positions and scales stem cell niches in plants. Sci. Adv. 2:e1500989. doi: 10.1126/sciadv.1500989

Jung, H. S., and Chory, J. (2010). Signaling between chloroplasts and the nucleus: can a systems biology approach bring clarity to a complex and highly regulated pathway? Plant Physiol. 152, 453-459. doi: 10.1104/pp.109.149070

Kleine, T., Maier, U. G., and Leister, D. (2009). DNA transfer from organelles to the nucleus: the idiosyncratic genetics of endosymbiosis. Annu. Rev. Plant Biol. 60, 115-138. doi: 10.1146/annurev.arplant.043008.092119

Kubalová, I., and Ikeda, Y. (2017). Chlorophyll measurement as a quantitative method for the assessment of cytokinin-induced green foci formation in tissue culture. J. Plant Growth Regul. 36, 516-521. doi: 10.1007/s00344-016-9637-7

Kubalová, I., Zalabák, D., Mičúchová, A., and Ikeda, Y. (2019). Mutations in tetrapyrrole biosynthesis pathway uncouple nuclear WUSCHEL expression from de novo shoot development in Arabidopsis. Plant Cell. Tissue Organ Cult. 139, 395-401. doi: 10.1007/s11240-019-01680-w

Kumar, A. M., and Söll, D. (2000). Antisense HEMA1 RNA expression inhibits heme and chlorophyll biosynthesis in Arabidopsis. Plant Physiol. 122, 49-56. doi: 10.1104/pp.122.1.49

Layer, G., Reichelt, J., Jahn, D., and Heinz, D. W. (2010). Structure and function of enzymes in heme biosynthesis. Protein Sci. 19, 1137-1161. doi: 10.1002/pro.405

Lopez-Juez, E., and Pyke, K. A. (2005). Plastids unleashed: their development and their integration in plant development. Int. J. Dev. Biol. 49, 557-577. doi: 10.1387/ijdb.051997el

Ma, F., Wang, L., Li, J., Samma, M. K., Xie, Y., Wang, R., et al. (2014). Interaction between $\mathrm{HY} 1$ and $\mathrm{H} 2 \mathrm{O} 2$ in auxin-induced lateral root formation in Arabidopsis. Plant Mol. Biol. 85, 49-61. doi: 10.1007/s11103-013-0168-3

Matsumoto, F., Obayashi, T., Sasaki-Sekimoto, Y., Ohta, H., Takamiya, K., and Masuda, T. (2004). Gene expression profiling of the tetrapyrrole metabolic pathway in Arabidopsis with a mini-array system. Plant Physiol. 135, 2379-2391. doi: $10.1104 /$ pp.104.042408

McCormac, A. C., Fischer, A., Kumar, A. M., Söll, D., and Terry, M. J. (2001). Regulation of HEMA1 expression by phytochrome and a plastid signal during de-etiolation in Arabidopsis thaliana. Plant J. 25, 549-561. doi: 10.1046/j.1365313x.2001.00986.x

Meng, W. J., Cheng, Z. J., Sang, Y. L., Zhang, M. M., Rong, X. F., Wang, Z. W., et al. (2017). Type-B Arabidopsis response regulators specify the shoot stem cell niche by dual regulation of WUSCHEL. Plant Cell 29, 1357-1372. doi: $10.1105 /$ tpc. 16.00640

Mense, S. M., and Zhang, L. (2006). Heme: a versatile signaling molecule controlling the activities of diverse regulators ranging from transcription factors to MAP kinases. Cell Res. 16, 681-692. doi: 10.1038/sj.cr.7310086

Miller, C. O., Skoog, F., Von Saltza, M. H., and Strong, F. M. (1955). Kinetin, a cell division factor from deoxyribonucleic acid. J. Am. Chem. Soc. 77:1392. doi: 10.1021/ja01610a105

Nishimura, C., Ohashi, Y., Sato, S., Kato, T., Tabata, S., and Ueguchi, C. (2004). Histidine kinase homologs that act as cytokinin receptors possess overlapping functions in the regulation of shoot and root growth in Arabidopsis. Plant Cell 16, 1365-1377. doi: 10.1105/tpc.021477

Papenbrock, J., Mock, H. P., Kruse, E., and Grimm, B. (1999). Expression studies in tetrapyrrole biosynthesis: inverse maxima of magnesium chelatase and ferrochelatase activity during cyclic photoperiods. Planta 208, 264-273. doi: 10.1007/s004250050558

Pernisova, M., Grochova, M., Konecny, T., Plackova, L., Harustiakova, D., Kakimoto, T., et al. (2018). Cytokinin signalling regulates organ identity via the AHK4 receptor in Arabidopsis. Development 145:dev163907. doi: 10.1242/dev. 163907

Pesaresi, P., Schneider, A., Kleine, T., and Leister, D. (2007). Interorganellar communication. Curr. Opin. Plant Biol. 10, 600-606. doi: 10.1016/j.pbi.2007. 07.007

Pontoppidan, B., and Kannangara, C. G. (1994). Purification and partial characterisation of barley glutamyl-tRNA(Glu) reductase, the enzyme that directs glutamate to chlorophyll biosynthesis. Eur. J. Biochem. 225, 529-537. doi: 10.1111/j.1432-1033.1994.00529.x

Richmond, A. E., and Lang, A. (1957). Effect of kinetin on protein content and survival of detached xanthium leaves. Science 125, 650-651. doi: 10.1126/ science.125.3249.650-a
Richter, A. S., Banse, C., and Grimm, B. (2019). The GluTR-binding protein is the heme-binding factor for feedback control of glutamyl-tRNA reductase. eLife 8, 1-18. doi: 10.7554/elife.46300

Riefler, M., Novak, O., Strnad, M., and Schmülling, T. (2006). Arabidopsis cytokinin receptor mutants reveal functions in shoot growth, leaf senescence, seed size, germination, root development, and cytokinin metabolism. Plant Cell 18, 40-54. doi: 10.1105/tpc.105.037796

Saeed, A. I., Sharov, V., White, J., Li, J., Liang, W., Bhagabati, N., et al. (2003). TM4: a free, open-source system for microarray data management and analysis. Biotechniques 34, 374-378. doi: 10.2144/03342mt01

Sakamoto, W., Miyagishima, S., and Jarvis, P. (2008). Chloroplast biogenesis: control of plastid development, protein import, division and inheritance. Arab. B. 6:e0110. doi: 10.1199/tab.0110

Shi, B., Guo, X., Wang, Y., Xiong, Y., Wang, J., Hayashi, K. I., et al. (2018). Feedback from lateral organs controls shoot apical meristem growth by modulating auxin transport. Dev. Cell 44, 204.e6-216.e6. doi: 10.1016/j.devcel.2017. 12.021

Shimizu, T., Yasuda, R., Mukai, Y., Tanoue, R., Shimada, T., Imamura, S., et al. (2020). Proteomic analysis of haem-binding protein from Arabidopsis thaliana and Cyanidioschyzon merolae. Philos. Trans. R. Soc. B Biol. Sci. 375:488. doi: 10.1098/rstb.2019.0488

Shin, J., Bae, S., and Seo, P. J. (2020). De novo shoot organogenesis during plant regeneration. J. Exp. Bot. 71, 63-72. doi: 10.1093/jxb/erz395

Skoog, F., and Miller, C. O. (1957). Chemical regulation of growth and organ formation in plant tissues cultured in vitro. Symp. Soc. Exp. Biol. 11, 118-130.

Snipes, S. A., Rodriguez, K., DeVries, A. E., Miyawaki, K. N., Perales, M., Xie, M., et al. (2018). Cytokinin stabilizes WUSCHEL by acting on the protein domains required for nuclear enrichment and transcription. PLoS Genet. 14:e1007351. doi: 10.1371/journal.pgen.1007351

Tanaka, R., Kobayashi, K., and Masuda, T. (2011). Tetrapyrrole metabolism in Arabidopsis thaliana. Arab. B. 9:e0145. doi: 10.1199/tab.0145

Tian, C., Wang, Y., Yu, H., He, J., Wang, J., Shi, B., et al. (2019). A gene expression map of shoot domains reveals regulatory mechanisms. Nat. Commun. 10, 1-12. doi: 10.1038/s41467-018-08083-z

Ueguchi, C., Sato, S., Kato, T., and Tabata, S. (2001). The AHK4 gene involved in the cytokinin-signaling pathway as a direct receptor molecule in Arabidopsis thaliana. Plant Cell Physiol. 42, 751-755. doi: 10.1093/pcp/pce094

Valvekens, D., Van Montagu, M., and Van Lijsebettens, M. (1988). Agrobacterium tumefaciens-mediated transformation of Arabidopsis thaliana root explants by using kanamycin selection. Proc. Natl. Acad. Sci. U.S.A. 85, 5536-5540. doi: 10.1073/pnas.85.15.5536

Von Gromoff, E. D., Alawady, A., Meinecke, L., Grimm, B., and Beck, C. F. (2008). Heme, a plastid-derived regulator of nuclear gene expression in Chlamydomonas. Plant Cell 20, 552-567. doi: 10.1105/tpc.107.05 4650

Xie, M., Chen, H., Huang, L., O’Neil, R. C., Shokhirev, M. N., and Ecker, J. R. (2018). A B-ARR-mediated cytokinin transcriptional network directs hormone crossregulation and shoot development. Nat. Commun. 9:1604. doi: 10.1038/s41467018-03921-6

$\mathrm{Xu}, \mathrm{C}$., and Hu, Y. (2020). The molecular regulation of cell pluripotency in plants. aBIOTECH 1, 169-177. doi: 10.1007/s42994-020-00028-9

Xuan, W., Zhu, F. Y., Xu, S., Huang, B. K., Ling, T. F., Qi, J. Y., et al. (2008). The heme oxygenase/carbon monoxide system is involved in the auxin-induced cucumber adventitious rooting process. Plant Physiol. 148, 881-893. doi: 10. 1104/pp.108.125567

Yadav, D., Zemach, H., Belausov, E., and Charuvi, D. (2019). Initial proplastid-tochloroplast differentiation in the developing vegetative shoot apical meristem of Arabidopsis. Biochem. Biophys. Res. Commun. 519, 391-395. doi: 10.1016/j. bbrc.2019.09.019

Yadav, R. K., Tavakkoli, M., Xie, M., Girke, T., and Venugopala, R. G. (2014). A high-resolution gene expression map of the Arabidopsis shoot meristem stem cell niche. Development 141, 2735-2744. doi: 10.1242/dev.10 6104

Zhai, Q., Li, C. B., Zheng, W., Wu, X., Zhao, J., Zhou, G., et al. (2007). Phytochrome chromophore deficiency leads to overproduction of jasmonic acid and elevated expression of jasmonate-responsive genes in Arabidopsis. Plant Cell Physiol. 48, 1061-1071. doi: 10.1093/pcp/pcm076 
Zhang, T.-Q., Lian, H., Zhou, C.-M., Xu, L., Jiao, Y., and Wang, J.-W. (2017). A Two-Step Model for de Novo Activation of WUSCHEL during Plant Shoot Regeneration. Plant Cell 29, 1073-1087. doi: 10.1105/tpc.16.00863

Zubo, Y. O., Blakley, I. C., Yamburenko, M. V., Worthen, J. M., Street, I. H., Franco-Zorrilla, J. M., et al. (2017). Cytokinin induces genome-wide binding of the type-B response regulator ARR10 to regulate growth and development in Arabidopsis. Proc. Natl. Acad. Sci. U.S.A. 114, E5995-E6004. doi: 10.1073/pnas. 1620749114

Zubo, Y. O., Yamburenko, M. V., Selivankina, S. Y., Shakirova, F. M., Avalbaev, A. M., Kudryakova, N. V., et al. (2008). Cytokinin stimulates chloroplast transcription in detached barley leaves. Plant Physiol. 148, 1082-1093. doi: 10.1104/pp.108.122275

Zürcher, E., Tavor-Deslex, D., Lituiev, D., Enkerli, K., Tarr, P. T., and Müller, B. (2013). A robust and sensitive synthetic sensor to monitor the transcriptional output of the cytokinin signaling network in planta. Plant Physiol. 161, 10661075. doi: $10.1104 /$ pp. 112.211763

Conflict of Interest: The authors declare that the research was conducted in the absence of any commercial or financial relationships that could be construed as a potential conflict of interest.

Copyright @ 2021 Ikeda, Zalabák, Kubalová, Králová, Brenner and Aida. This is an open-access article distributed under the terms of the Creative Commons Attribution License (CC BY). The use, distribution or reproduction in other forums is permitted, provided the original author(s) and the copyright owner(s) are credited and that the original publication in this journal is cited, in accordance with accepted academic practice. No use, distribution or reproduction is permitted which does not comply with these terms. 